\title{
Avaliação dos resultados de análises de Escherichia coli para verificação do controle de processos em um estabelecimento de abate de bovinos*
}

\section{Evaluation of Escherichia coli test results for process control verification at a bovine slaughterhouse}

\author{
Leandro Casagrande, ${ }^{* *}$ Camila Menegon Teixeira Detanico, ${ }^{* *}$ Robson Maia Franco***
}

\begin{abstract}
Resumo
O objetivo do presente estudo foi comparar diferentes critérios de classificação e interpretação de análises de Escherichia coli genérica, utilizadas na verificação de controles de processos de abate. Utilizaram-se três critérios para a classificação de 1111 análises de superfície de carcaças bovinas: aceitáveis (negativas); marginais (positivas porém inferiores a um limite superior); e inaceitáveis (acima do limite superior). Como limite superior adotaram-se parâmetros estabelecidos pelas legislações americana (100 UFC/ $\mathrm{cm}^{2}$ ) e australiana (20 UFC/ $\mathrm{cm}^{2}$ ), e dois valores obtidos da média mais duas vezes o desvio padrão dos 1111 resultados $\left(5,31 \mathrm{UFC} / \mathrm{cm}^{2}\right)$ e apenas dos 49 resultados positivos $\left(27,43 \mathrm{UFC} / \mathrm{cm}^{2}\right)$. Para a avaliação das análises, foram comparadas duas estratégias de janela móvel, com limites para a ocorrência de resultados marginais e inaceitáveis. Houve diferença significativa apenas quando utilizados o menor e o maior limites superiores. Com o limite de $5,31 \mathrm{UFC} / \mathrm{cm}^{2}$ o número de resultados inaceitáveis $(16,32 \%)$ foi superior ao esperado estatisticamente. Com $100 \mathrm{UFC} / \mathrm{cm}^{2}$, observaram-se apenas resultados aceitáveis. Essa variação demonstra que o uso de um limite inadequado poderia levar a tomada de medidas corretivas excessivas ou insuficientes, prejudicando o controle de processos. Contudo, quando os resultados foram aplicados às duas metodologias de janela móvel, não foram observadas diferenças significativas quanto ao número de ações corretivas a serem tomadas. Portanto, a média da contagem de E. coli calculada em cada estabelecimento, obtida somente dos resultados positivos, somada ao uso de uma janela móvel, mostra-se a ferramenta mais aplicável para um diagnóstico contínuo e manutenção do controle de processos de abate.
\end{abstract}

Palavras-chave: análise bacteriológica, carcaça bovina, Escherichia coli genérica.

\begin{abstract}
Escherichia coli tests are used to verify the adequacy of slaughter process controls whit respect to fecal contamination. This research aimed to evaluate different performance criteria for generic $E$. coli test results, in 1111 swab samples of beef carcasses. Results were classified as acceptable, marginal and unacceptable by the American and Australian standards, 100 and 20 CFU/ $\mathrm{cm}^{2}$ respectively, and two parameters calculated according Brazilian guidelines considering the mean plus two standard deviations: $5.31 \mathrm{CFU} / \mathrm{cm}^{2}$, obtained from the total of results; and $27.43 \mathrm{CFU} / \mathrm{cm}^{2}$ considering only positive samples. Only the limits of 5,31 and $100 \mathrm{CFU} / \mathrm{cm}^{2}$ did not present the expected distribution of marginal and unacceptable results $(83.68 \%$ and $16.32 \%$; and $100 \%$ and $0 \%$, respectively), and could lead to excessive or insufficient corrective actions to prevent fecal contamination. The evaluation of E. coli results were performed using two strategies of a moving window approach, considering 13 or 15 consecutive samples and limits on occurrence of marginal and unacceptable results. No significant differences were observed in the number of necessary corrective actions, which indicates that moving window approach considers mainly the occurrence of $E$. coli and not only high bacteria counts. Thus, the mean count only of positive results of $E$. coli calculated in each establishment applied in a moving window considering the occurrence of bacteria was the most applicable instrument to identify failures during production, allowing an effective slaughter process control and pathogen reduction.
\end{abstract}

Keywords: bacterial analysis, beef carcass, generic Escherichia coli.

\footnotetext{
*Recebido em 11 de julho de 2012 e aceito em 13 de maio de 2013.

${ }^{* *}$ Ministério da Agricultura, Pecuária e Abastecimento (MAPA), Serviço de Inspeção Federal, Rodovia MT 358, Km 05, s/n, 78300-000, Tangará da Serra, MT, Brasil.

***Universidade Federal Fluminense (UFF), Faculdade de Veterinária, Departamento de Tecnologia dos Alimentos, Niterói, RJ, Brasil.

Autor para correspondência: Leandro Casagrande. E-mail: leandro.casagrande@agricultura.gov.br
} 


\section{Introdução}

A detecção de Escherichia coli em superfície de carcaças bovinas está associada à ocorrência de contaminação fecal durante o seu processamento como resultado de possíveis falhas operacionais. Logo, os resultados das análises deste micro-organismo são importantes ferramentas de mensuração da qualidade higiênica dos procedimentos realizados nas indústrias de abate (Tergney e Bolton, 2006).

A pesquisa de $E$. coli genérica - biótipo I em carcaças bovinas vem sendo utilizada como instrumento de verificação do controle higiênico-sanitário de processos desde 1996, quando passou a ser exigida nos Estados Unidos pelo Serviço de Inspeção e Segurança dos Alimentos (Food Safety and Inspection Service FSIS) como parte de seu programa de redução de patógenos. A partir do levantamento de dados nacionais, o FSIS estabeleceu valores de referência e critérios para a classificação das análises de $E$. coli em aceitáveis, marginais e inaceitáveis de acordo com a contagem deste micro-organismo, bem como sua avaliação, permitindo assim a identificação de eventuais desvios e a adoção de medidas corretivas para a manutenção do controle dos processos (USDA, 1996a). O Serviço de Inspeção Australiano (Australian Quarantine and Inspection Service - AQIS) também realiza o monitoramento de microorganismos em indústrias de abate. Desde 1997, análises de E. coli e de Salmonella spp são realizadas como parte de um programa nacional para identificação de desvios e manutenção do controle operacional das indústrias em relação à contaminação fecal (AQIS, 2003).

No Brasil, os testes rotineiros para $E$. coligenérica foram instituídos para estabelecimentos exportadores em janeiro de 1997 pelo Ministério da Agricultura, Pecuária e Abastecimento (MAPA) mantendo-se o estabelecido pela legislação americana (Brasil, 1996). Entretanto, em dezembro de 1997, a categorização dos resultados foi substituída pela análise estatística dos mesmos, realizada a partir de registros feitos em cada estabelecimento com a determinação de médias e limites próprios para a identificação de tendências (Brasil, 1997).

Desde então, não há no Brasil valores de referência para a análise dos resultados dos testes de $E$. coli genérica, devendo cada estabelecimento determinar os seus parâmetros a partir de uma série histórica de dados (Brasil, 2006). A obtenção desses critérios, contudo, não foi claramente descrita nas instruções publicadas pelo MAPA, havendo grande divergência nos valores utilizados como limites, bem como na interpretação dos resultados das análises microbiológicas por parte das indústrias de abate de bovinos e, portanto, há diferenças na tomada de ações preventivas e corretivas entre os estabelecimentos.

Assim, o presente estudo comparou diferentes metodologias para a avaliação e interpretação dos resultados de E. coli genérica em superfície de carcaças bovinas a fim de determinar a forma mais adequada para o seu gerenciamento em um estabelecimento de abate.

\section{Material e métodos}

Foram avaliados os resultados de análises referentes a 1111 amostras de suabe de superfície de meias carcaças bovinas obtidas em um matadouro-frigorífico sob Inspeção Federal, localizado no Estado de Mato Grosso, habilitado à exportação, entre os meses de janeiro e dezembro de 2010.
As amostras foram coletadas em três pontos distintos de meias carcaças resfriadas, sorteadas aleatoriamente, aproximadamente 24 horas após a matança, numa frequência de um teste a cada 300 carcaças. Os pontos de coleta foram realizados, nesta sequência, nas regiões do vazio, do peito e da alcatra. Estes foram demarcados por moldes metálicos estéreis com área de $100 \mathrm{~cm}^{2}$ e para cada região realizou-se a aplicação de suabes, previamente umedecidos em $10 \mathrm{ml}$ de solução salina peptonada tamponada $1 \%$, por 10 vezes no sentido vertical e por 10 vezes no horizontal (Brasil, 1997). Os suabes acondicionados em sacos estéreis foram armazenados em caixas isotérmicas e transportados ao laboratório credenciado pelo MAPA onde foram analisados pela metodologia AOAC $998.08-$ E. coli Petrifilm ${ }^{\mathrm{TM}}$ (Horwitz e Latimer, 2005), validada pelo MAPA (Brasil, 2005). Foram inoculadas alíquotas de $1 \mathrm{ml}$ das amostras em placas Petrifilm ${ }^{\mathrm{TM}}$ EC para a identificação e contagem do número de colônias. Após incubação por $24 \pm 1 \mathrm{~h}$ à $35 \pm 2^{\circ} \mathrm{C}$, aquelas que apresentaram coloração azul com produção de gás foram quantificadas como $E$. coli. Os resultados foram expressos em unidades formadoras de colônia (UFC) por unidade de área $\left(\mathrm{cm}^{2}\right.$ ), sendo o limite de detecção 0,083 UFC/cm² (Horwitz e Latimer, 2005). Valores abaixo deste limite foram considerados resultados negativos.

Os resultados foram então classificados em três categorias segundo os valores das contagens de E.coli em UFC/cm², sendo considerados aceitáveis, os resultados negativos; marginais, os resultados com valores entre o limite de detecção $(0,083$ UFC/ $\mathrm{cm}^{2}$ ) e o limite superior; e inaceitáveis, aqueles acima do limite superior (USDA, 1996a; Brasil, 1997). Utilizaram-se quatro limites superiores distintos para a definição das categorias: dois limites superiores estabelecidos pelo FSIS e pelo serviço de inspeção australiano (Australian Quarentine Inspection Service AQIS), sendo eles $100 \mathrm{UFC} / \mathrm{cm}^{2}$ e $20 \mathrm{UFC} / \mathrm{cm}^{2}$, respectivamente (USDA, 1996a; AQIS, 2003); e dois limites superiores calculados a partir da média dos valores das contagens de E. coli em UFC/ $\mathrm{cm}^{2}$ dos resultados, conforme o método de Análise Estatística de Processo (Brasil, 1997). O primeiro limite calculado foi obtido a partir da contagem total do microorganismo durante o período de análise, incluindo os negativos, método que é realizado atualmente por diversos estabelecimentos. O segundo limite calculado foi obtido apenas dos resultados positivos, ambos somados a duas vezes o desvio padrão, conforme orientações da legislação brasileira (Brasil, 2008).

Para a avaliação das análises de E. coli foram comparadas duas abordagens de janela móvel que consideram a ocorrência de resultados marginais e inaceitáveis em um número determinado de amostras para a identificação de desvios. Com a primeira janela móvel, descrita pelo FSIS, foram analisados sempre os últimos 13 resultados na ordem em que foram obtidos. A inclusão de um novo resultado excluía o primeiro, e assim sucessivamente (USDA, 1996a). Com a segunda janela móvel, baseada no sistema australiano, foram avaliados 15 resultados consecutivos (AQIS, 2003). Nos dois casos, os resultados da avaliação por meio da janela móvel foram considerados "reprovados" na ocorrência de mais de três resultados marginais ou um resultado inaceitável. Ocorrendo até três resultados marginais e nenhum resultado inaceitável, os resultados da avaliação foram considerados "aprovados". Considerando a metodologia australiana, a cada resultado reprovado encontrado, a contagem das próximas 15 amostras era reiniciada mesmo 
que a janela ainda não tivesse sido completada, diferentemente do método americano que mantém uma janela contínua de 13 amostras.

As porcentagens dos resultados em cada categoria foram comparadas pelo teste qui-quadrado $(p<0,05)$, utilizando-se $o$ programa SPSS Statistics 17.0. As médias e desvios padrão foram obtidos no programa SigmaStat 3.5.

\section{Resultados e discussão}

Das 1111 amostras analisadas, 1062 (95,6\%) apresentaram resultados negativos para a presença de $E$. coli genérica e foram consideradas aceitáveis. As demais 49 análises (4,4\%) apresentaram resultados positivos, com contagens variando entre $0,083 \mathrm{UFC} / \mathrm{cm}^{2}$ e $77,083 \mathrm{UFC} / \mathrm{cm}^{2}$ e foram classificadas como marginais ou inaceitáveis de acordo com cada um dos quatro limites superiores. A média da contagem total do microorganismo obtida das 1111 análises foi de $0,18 \pm 2,56$ $\mathrm{UFC} / \mathrm{cm}^{2}$, resultando em um limite superior de $5,31 \mathrm{UFC} / \mathrm{cm}^{2}$. Em relação ao cálculo do limite superior obtido somente dos resultados positivos (49 análises), obteve-se uma média de 4,08 $\pm 11,67$ UFC/cm² e um limite superior de 27,43 UFC/cm². A distribuição dos resultados positivos está apresentada na tabela 1.

Tabela 1: Classificação dos resultados positivos de E. coli genérica em superfície de carcaças bovinas

\begin{tabular}{lcccc}
\hline & \multicolumn{4}{c}{ Limites superiores $\left(\mathrm{UFC} / \mathrm{cm}^{2}\right)$} \\
\cline { 2 - 5 } Resultados & FSIS & AQIS & TOTAL & POSITIVOS \\
& 100 & 20 & 5,31 & 27,43 \\
\hline Marginais & $49(100 \%)^{*}$ & $47(95,92 \%)$ & $41(83,68 \%)^{*}$ & $48(97,96 \%)$ \\
Inaceitáveis & $0(0 \%)^{*}$ & $2(4,08 \%)$ & $8(16,32 \%)^{*}$ & $1(2,04 \%)$
\end{tabular}

* valores observados desviam significativamente dos valores esperados no teste qui-quadrado $(p=0,002)$.

Apenas as distribuições em torno do limite superior de 20 UFC/ $/ \mathrm{cm}^{2}$, instituído pelo AQIS, (95,92\% marginais e 4,08\% inaceitáveis) e do limite superior de $27,43 \mathrm{UFC} / \mathrm{cm}^{2}$ ), definido a partir da média dos resultados positivos, $(97,96 \%$ marginais e $2,04 \%$ inaceitáveis) apresentaram uma proporção de resultados marginais e inaceitáveis dentro do esperado estatisticamente. Com o limite de $5,31 \mathrm{UFC} / \mathrm{cm}^{2}$ observaram-se números inferiores de resultados marginais $(83,68 \%)$ e superiores de resultados inaceitáveis $(16,32 \%)$ em relação ao esperado. Com o limite de $100 \mathrm{UFC} / \mathrm{cm}^{2}$, nenhum resultado inaceitável foi observado (Tabela 1).

As diferenças observadas na categorização dos resultados evidenciam a importância da correta determinação dos limites superiores a serem utilizados, pois os resultados microbiológicos terão impacto direto nas ações a serem tomadas pela Inspeção Federal e pelo controle de qualidade das empresas a fim de monitorar e manter sob controle os processos de abate. Entretanto, a legislação brasileira não apresenta um limite predeterminado para a classificação dos resultados de E. coli genérica em superfície de carcaças bovinas.
Conforme o disposto na Circular n 835 da Coordenação Geral de Programas Especiais (CGPE) do MAPA, o limite superior é definido como a média de uma série histórica de resultados somado duas vezes o desvio padrão, e não há instruções sobre de que forma essa média deve ser obtida ou quais resultados devem ser utilizados na sua determinação. Além disso, a aplicação de medidas corretivas e preventivas deve ocorrer apenas quando o limite superior for extrapolado (Brasil, 2006). Assim, a falta de padronização na avaliação dos dados pode resultar em ações de controle equivocadas, desnecessárias ou insuficientes. O uso das duas médias calculadas de maneiras diferentes neste estudo mostrou uma variação na classificação das análises microbiológicas (Tabela 1), e como cada distribuição, em função dos limites escolhidos, pode representar dois cenários distintos: um demonstrando que os processos industriais estão sob controle e o outro demonstrando a falta de controle higiênico-sanitário das operações, apesar dos resultados em UFC/ $\mathrm{cm}^{2}$ serem os mesmos.

Muitos estabelecimentos vêm utilizando a média do total de resultados de $E$. coli genérica (positivos e negativos) para estabelecer o limite que irá classificá-los. Porém, assumir os resultados negativos na determinação da média de contagem deste microorganismo pode resultar em um valor inferior do que o realmente detectado. A média do total de resultados calculada anteriormente, mesmo com a soma de dois desvios, classifica $16,32 \%$ dos resultados como inaceitáveis, um número aproximadamente oito vezes maior do que o obtido com o limite calculado apenas a partir dos resultados positivos $(2,04 \%)$, o que resultaria, portanto, na tomada excessiva de ações.

Em um estabelecimento com baixa ocorrência de $E$. coli, a exemplo o estabelecimento pesquisado (4,4\% de presença), a porcentagem de resultados negativos é alta, resultando em uma distribuição assimétrica dos dados, ou seja, os valores não se distribuem igualmente ao redor da média. Para avaliação estatística nessas situações, o uso de outras medidas, tais como mediana, quartil e percentil, pode refletir melhor as características da distribuição dos resultados do que a média (Callegari-Jacques, 2003). Em um estudo para estimar a prevalência de microorganismos de interesse à saúde coletiva nos Estados Unidos, de 2112 amostras analisadas, 1779 foram negativas $(84,2 \%)$ (USDA, 1996b). Com base nesses dados, os critérios de verificação estabelecidos pelo FSIS foram determinados a partir dos valores correspondentes ao $80^{\circ}$ e ao $98^{\circ}$ percentil (5 UFC/ $\mathrm{cm}^{2}$, limite de detecção do ensaio, e 100 UFC $/ \mathrm{cm}^{2}$, respectivamente): resultados aceitáveis, aqueles abaixo do $80^{\circ}$ percentil; marginais entre o $80^{\circ}$ e o $98^{\circ}$ percentil; e inaceitáveis os acima do $98^{\circ}$ percentil (USDA, 1996a).

No Brasil, segundo a Circular $n^{\circ} 273$, do Departamento de Inspeção de Produtos de Origem Animal - DIPOA/MAPA, de 22 de dezembro de 1997, esses valores de referência e a categorização dos resultados deixaram de ser utilizados devido à falta de um fator de conversão que possibilitasse a correlação dos resultados obtidos com o método de suabe (não-destrutivo), adotado no país, com aqueles obtidos pelo método de excisão de tecido (destrutivo), utilizado no estudo americano (Brasil, 1997). O FSIS, contudo, orienta a utilização desses parâmetros mesmo em casos de coleta não-destrutiva, pois além de evitar danos à superfície das carcaças, espera-se que a maior área amostrada, cinco vezes maior na técnica de suabe $\left(300 \mathrm{~cm}^{2}\right)$ em relação a metodologia destrutiva $\left(60 \mathrm{~cm}^{2}\right)$, forneça resultados comparáveis aos da técnica de excisão (USDA, 1996a). 
Alguns estudos já demonstraram a relação positiva entre área amostrada e a detecção de microorganismos. Pearce e Bolton (2005) observaram um maior número de amostras positivas para enterobactérias em carcaças bovinas ao utilizar o método de esponja em relação ao método de excisão, atribuindo o resultado à diferença da área de amostragem (100 $\mathrm{cm}^{2}$ para esponja e $5 \mathrm{~cm}^{2}$ para excisão). Gill e Jones (2000) demonstraram que para bactérias com baixa freqüência em carcaças, como as enterobactérias, as chances de coletá-las dobram para cada aumento em dez vezes da área amostrada. Segundo os resultados de Ghafir e Daube (2008), a coleta não destrutiva abrangendo uma área total de $600 \mathrm{~cm}^{2}$ em meias carcaças suínas mostrou-se eficiente na determinação de contagens de $E$. coli em comparação ao método de excisão de referência, que compreendia $20 \mathrm{~cm}^{2}$.

Esses estudos, embora não estabeleçam uma correlação direta entre as duas metodologias, têm demonstrado que a coleta não destrutiva é adequada para o uso em estabelecimentos de abate na verificação de controle de processos (Martínez et al., 2010).

O FSIS manteve os critérios para a categorização e avaliação das análises de $E$. coli estabelecidos em 1996 mesmo após a divulgação de novos resultados obtidos pelo método não destrutivo em 2005 (USDA, 2005). Apesar da diferença encontrada na média de contagem de E.coli (33 UFC/ $\mathrm{cm}^{2}$ no primeiro estudo e $0,26 \mathrm{UFC} / \mathrm{cm}^{2}$ no segundo), as ocorrências foram semelhantes, $15,8 \%$ no primeiro estudo e $16,6 \%$ no estudo seguinte, indicando que a detecção deste microorganismo não foi comprometida pelo método de coleta utilizado (Eblen et al., 2005).

A metodologia para a interpretação dos resultados bacteriológicos atualmente utilizada no Brasil não considera a ocorrência de $E$. coli genérica para a adoção de medidas corretivas (Brasil, 2006), mas sim as contagens, que podem ser bastante variáveis. Assim, por não haver um valor de referência nacional a interpretação torna-se dificultada. Barros et al. (2007), ao pesquisarem onze estabelecimentos no Estado do Paraná, encontraram uma média de contagem de $E$. coli de $10,23 \mathrm{UFC} / \mathrm{cm}^{2} \mathrm{com}$ variação de $0,89 \mathrm{UFC} / \mathrm{cm}^{2}$ a $3019,95 \mathrm{UFC} / \mathrm{cm}^{2}$, valores superiores ao demonstrado no presente estudo. Por outro lado, resultados de Caselani (2010) em pesquisa realizada em um abatedouro no Estado de São Paulo demonstraram uma média inferior ao presente estudo, sendo esta de 1,62 $\pm 3,23 \mathrm{UFC} / \mathrm{cm}^{2}$. Entretanto, a ocorrência foi de $22 \%$, variando entre os meses do ano e de acordo com o sistema de criação dos animais.

Dessa forma, limitar a adoção de medidas corretivas apenas quando há detecção de contagens acima de determinado limite superior pode comprometer o controle das operações, pois as possíveis variações na ocorrência de $E$. coli genérica não são consideradas. Além disso, desconsiderar a ocorrência de resultados marginais também pode ocultar as falhas ocorridas durante as operações. A categorização das análises microbiológicas com aplicação de limites para a ocorrência de resultados marginais e inaceitáveis é a estratégia adotada em países como Estados Unidos e Austrália para o acompanhamento do desempenho dos processos de abate. Além disso, os limites são aplicados na medida em que os resultados são obtidos, permitindo uma aplicabilidade mais imediata das análises microbiológicas como ferramentas de verificação e monitoramento de processo (AQIS, 2003; USDA, 1996a). As duas abordagens de janela móvel, americana e australiana, foram aplicadas às 1111 análises de $E$. coli obtidas em 2010 (Tabela 2).
Tabela 2: Distribuição dos resultados de E. coli genérica em superfície de carcaças bovinas após aplicação de uma janela móvel de 13 e 15 amostras

\begin{tabular}{|c|c|c|c|c|c|}
\hline \multirow{3}{*}{ Janela Móvel } & \multirow{3}{*}{ Resultados } & \multicolumn{4}{|c|}{ Limites superiores (UFC/cm²) } \\
\hline & & FSIS & AQIS & TOTAL & POSITIVOS \\
\hline & & 100 & 20 & 5,31 & 27,43 \\
\hline \multirow{2}{*}{$\begin{array}{c}13 \text { amostras } \\
\text { FSIS }\end{array}$} & Aprovados & $1106(99,55 \%)$ & $1105(99,45 \%)$ & 1100 (99\%) & $1105(99,45 \%)$ \\
\hline & Reprovados & $5(0,45 \%)$ & $6(0,55 \%)$ & $11(1 \%)$ & $6(0,55 \%)$ \\
\hline \multirow{2}{*}{$\begin{array}{c}15 \text { amostras } \\
\text { AQIS }\end{array}$} & Aprovados & $1109(99,8 \%)$ & $1108(99,7 \%)$ & $1103(99,3 \%)$ & $1108(99,7 \%)$ \\
\hline & Reprovados & $2(0,02 \%)$ & $3(0,3 \%)$ & $8(0,7 \%)$ & $3(0,3 \%)$ \\
\hline
\end{tabular}

Não foram observadas diferenças significativas quando comparados os resultados aprovados e reprovados para os quatro limites superiores utilizados, indicando que com esta metodologia, o que realmente influencia no resultado é a ocorrência do microorganismo e não os valores das contagens. Contudo, na prática, uma grande diferença é identificada. No sistema americano, utilizando-se o menor limite superior $(5,31$ UFC $/ \mathrm{cm}^{2}$ ) seriam necessárias 11 intervenções a fim de se identificar e corrigir possíveis falhas no controle de processos. Com os demais limites $\left(20,27,43\right.$ e $\left.100 \mathrm{UFC} / \mathrm{cm}^{2}\right)$ o número de ações reduziria em aproximadamente $50 \%(6,6$ e 5 , respectivamente). Com a aplicação da janela móvel australiana, o número de intervenções seria ainda menor: 8 para o limite de $5,31 \mathrm{UFC} / \mathrm{cm}^{2}$; 3 para os limites de 20 e $27,43 \mathrm{UFC} / \mathrm{cm}^{2}$; e 2 para o limite de $100 \mathrm{UFC} / \mathrm{cm}^{2}$.

A escolha da melhor abordagem poderia, portanto, ser definida em função da ocorrência do microorganismo em cada estabelecimento. Quando a ocorrência de $E$. coli é alta, a janela de 13 amostras parece ser a mais aplicável, visto que o número de intervenções será maior. Neste caso, cada resultado positivo, mesmo que não seja inaceitável, contribui para a alta ocorrência do microorganismo, logo precisa ser considerado e avaliado de forma a permitir a correção de possíveis falhas operacionais. No sistema americano, cada novo resultado reprovado dispara uma ação buscando identificar e solucionar possíveis falhas no processo. Dessa forma, pode ser tomada mais de uma ação sobre um mesmo resultado reprovado, o que seria importante para a redução de uma alta ocorrência do microorganismo, como as observadas nos Estados Unidos nos anos de 1996 e 1997 (Eblen et al., 2005).

Em uma situação de baixa prevalência, como o registrado na Austrália em 2003, 3\% a 7\% (Kiermeier et al., 2006), presume- 
se que os processos já estejam sob controle, sendo necessária apenas a manutenção. Portanto, a janela móvel de 15 amostras com o reinicio da contagem pode ser a mais eficiente, pois o foco desta abordagem está na detecção de eventuais desvios. Nesta metodologia, ao reiniciar a contagem na ocorrência de um resultado reprovado, as ações não incidem mais de uma vez sobre cada um dos resultados.

\section{Conclusão}

O uso de uma média histórica obtida em cada estabelecimento, considerando suas particularidades, calculada apenas a partir de resultados positivos de $E$. coli mostrou-se ser o critério mais adequado para a classificação das análises em três categorias:

\section{Referências}

AUSTRALIAN QUARANTINE AND INSPECTION SERVICE. AQIS Notice Meat 2003/6. 2003. Disponível em: <http://www.daff.gov. au/aqis/export/meat/elmer-3/notices/archived_meat_notices $>$. Acesso em: 18 ago. 2011.

BARROS, M.F.A.; NERO, L.A.; MONTEIRO, A.A.; BELOTI, V. Identification of main contamination points by hygiene indicator microorganisms in beef processing plants. Ciência e Tecnologia de Alimentos, v.27, n.4, p.856-862, 2007. Disponível em: <http:// dx.doi.org/10.1590/S0101-20612007000400028>. Acesso em: 9 set. 2011.

BRASIL. Ministério da Agricultura e do Abastecimento. Secretaria de Defesa Agropecuária. Departamento de Inspeção de Produtos de Origem Animal. Circular $n^{\circ}$ 245/96/DCI/DIPOA, de 25 de novembro de 1996. Dispõe sobre a exportação de carnes e produtos cárneos para os EUA. Brasília, DF, 25 nov. 1996.

BRASIL. Ministério da Agricultura e do Abastecimento. Secretaria de Defesa Agropecuária. Departamento de Inspeção de Produtos de Origem Animal. Circular $n^{\circ}$ 273/97/DIPOA, de 22 de dezembro de 1997. Dispõe sobre a exportação de produtos à base de carne para os EUA. Brasília, DF, 22 dez. 1997.

BRASIL. Ministério da Agricultura, Pecuária e Abastecimento. Instrução Normativa $n^{\circ} 40$, de 12 de dezembro de 2005. Aprova métodos analíticos para análises microbiológicas de produtos de origem animal. Diário Oficial da União, Brasília, DF, 16 dez. 2005. Seção 1.

BRASIL. Ministério da Agricultura, Pecuária e Abastecimento. Secretaria de Defesa Agropecuária. Departamento de Inspeção de Produtos de Origem Animal. Coordenação Geral de Programas Especiais. Circular $n^{\circ}$ 835/2006/CGPE/DIPOA, de 13 de novembro de 2006. Dispõe sobre testes microbiológicos em carcaças de bovinos. Brasília, DF, 13 nov. 2006.

BRASIL. Ministério da Agricultura, Pecuária e Abastecimento. Secretaria de Defesa Agropecuária. Departamento de Inspeção de Produtos de Origem Animal. Coordenação Geral de Programas Especiais. Circular $n^{\circ}$ 1058/2008/CGPE/DIPOA, de 13 de novembro de 2008. Dispõe sobre a interpretação dos resultados de E. coli. Brasília, DF, 13 nov. 2008.

CALLEGARI-JACQUES, S.M. Bioestatística: princípios e aplicações. Porto Alegre: Artmed, 2003. 255p.

CASELANI, K. Avaliação dos controles microbiológicos e do programa de redução de patógenos no abate de bovinos. 2010. 123 f. Dissertação (Mestrado) - Faculdade de Ciências Agrárias e Veterinárias - Universidade Estadual Paulista Júlio de Mesquita Filho, Jaboticabal, 2010. aceitáveis (resultados negativos), marginais (resultados positivos entre o limite de detecção da análise microbiológica e o limite superior obtido da média mais duas vezes o desvio padrão dos resultados positivos) e inaceitáveis (resultados acima do limite superior estabelecido). Concluiu-se ainda que a essa categorização dos resultados, somada a análise por meio de uma janela móvel modulada à ocorrência deste microorganismo é a ferramenta mais aplicável para o acompanhamento dos processos de abate. Neste contexto, todo resultado positivo é considerado e não apenas as altas contagens do microorganismo Além disso, o ciclo de amostras analisadas pode ser completado em pouco tempo, permitindo ações mais rápidas e, portanto, a manutenção contínua do controle dos processos em um efetivo programa de redução de patógenos.

EBLEN, D.R.; LEVINE, P.; ROSE, B.E.; SAINI, P.; MAGEAU, R.; HILL, W.E. Nationwide Microbiological Baseline Data Collected by Sponge Sampling during 1997 and 1998 for Cattle, Swine, Turkeys, and Geese. Journal of Food Protection, v.68, n.9, p.18481852, 2005.

GHAFIR, Y.; DAUBE, G. Comparison of swabbing and destructive methods for microbiological pig carcass sampling. Letters in Applied Microbiology, v.42, p.322-326, 2008. Disponível em: <http:// onlinelibrary.wiley.com/doi/10.1111/j.1472-765X.2008.02433.x/ pdf>. Acesso em: 12 jan. 2012.

GILL, C.O.; JONES, T. Microbiological sampling of carcasses by excision or swabbing. Journal of Food Protection, v.63, n.2, p.16773, 2000.

HORWITZ, W.; LATIMER, G.W. (Eds.). Official Methods of Analysis of AOAC International. 18th ed. Gaithersburg: AOAC International, 2005.

KIERMEIER, A.; BOBBITT, J.; VANDERLINDE, P.; HIGGS, G.; POINTON.; SUMNER, J. Use of routine beef carcass Escherichia coli monitoring data to investigate the relationship between hygiene status of incoming stock and processing efficacy. International Journal of Food Microbiology, v. 111, p. 263-269, 2006.

MARTÍNEZ, B.; CELDA, M.F.; ANASTASIO, B.; GARCÍA, I.; LÓPEZ-MENDOZA, M.C. Microbiological sampling of carcasses by excision or swabbing with three types of sponge or gauze. Journal of Food Protection, v.73, n.1, p.81-87, 2010.

PEARCE, R.A; BOLTON, D.J. Excision vs sponge swabbing: a comparison of methods for the microbiological sampling of beef, pork and lamb carcasses. Journal of Applied Microbiology, v.98, p.896-900, 2005. Disponível em: <http://onlinelibrary.wiley.com/ doi/10.1111/j.1365-2672.2004.02525.x/pdf>. Acesso em: 13 jan. 2012.

TERGNEY, A.; BOLTON, D.J. Validation studies on an online monitoring system for reducing faecal and microbial contamination on beef carcasses. Food Control, v. 17, p. 378-382, 2006.

UNITED STATES DEPARTMENT OF AGRICULTURE. Food Safety and Inspection Service. Pathogen Reduction; Hazard Analysis and Critical Control Point (HACCP) Systems; Final Rule. Federal Register, Washington, DC, v.61, n.144, p.38805, 25 jul. 1996a.

UNITED STATES DEPARTMENT OF AGRICULTURE. Food Safety and Inspection Service. Nationwide beef microbiological baseline data collection program: cows and bulls - December 1993 - November 1994. 1996b. Disponível em: <http://www.fsis.usda. gov/Science/Baseline_Data/index.asp>. Acesso em: 29 ago. 2011. UNITED STATES DEPARTMENT OF AGRICULTURE. Food Safety and Inspection Service. Generic E. coli and Salmonella results. Federal Register, Washington, DC, v. 70, n. 32, p. 80588060, 17 dez. 2005. 